\title{
Hospitalizações por infarto agudo do miocárdio segundo o dia da semana: estudo retrospectivo
} Myocardial infarction hospitalization by the day of the week: retrospective study

Juan S Yazlle Rocha e G leiton C M Silva

Departamento de Medicina Social da Faculdade de Medicina de Ribeirão Preto da Universidade de São Paulo. Ribeirão Preto, SP, Brasil 


\title{
Hospitalizações por infarto agudo do miocárdio segundo o dia da semana: estudo retrospectivo* Myocardial infarction hospitalization by the day of the week: retrospective study
}

\author{
Juan S Yazlle Rocha e Gleiton C M Silva
}

Departamento de Medicina Social da Faculdade de Medicina de Ribeirão Preto da Universidade de São Paulo. Ribeirão Preto, SP, Brasil

\section{D escritores}

Hospitalização, estatística\#. Infarto do miocárdio, epidemiologia”. Classe social" . Inequidade na saúde". Sistemas pré-pagos de saúde. Hospitais privados. SUS (Br).

\section{Keywords}

Hospitalization, statistic ${ }^{\#}$.

Myocardial infarction,

epidemiology". Social class ${ }^{\#}$.

Health inequity".

\section{Resumo}

\section{Objetivo}

A literatura tem relatado a associação do infarto com a segunda-feira, sugerindo a existência de mecanismos de "gatilho" no desencadeamento da doença. Foi realizado estudo para verificar a distribuição semanal das hospitalizações por infarto do miocárdio e sua associação ao sexo e à categoria da internação (condição social) na região de Ribeirão Preto de 1987 a 1996.

Métodos

Foram estudadas 173.982 hospitalizações por doenças cardíacas e vasculares cerebrais, incluindo 5.804 casos de infarto agudo do miocárdio, referidos a um centro de registro contínuo de informações de assistência hospitalar. As hospitalizações foram classificadas em particulares, de sistemas privados de pré-pagamento e do Sistema Único de Saúde (SUS). Foram estudadas as associações com o sexo, a condição do egresso e a categoria da internação.

Resultados/Conclusões

A distribuição semanal de hospitalizações por doenças cardiovasculares e infarto agudo do miocárdio apresentou um pico às segundas-feiras: $19,3 \%$ e $16,9 \%$ dos casos, respectivamente. Houve queda acentuada no final de semana $(8,6 \%)$ dos casos de doenças cardiovasculares e menor redução dos casos de infarto agudo do miocárdio $(12,7 \%)$, sendo essas diferenças estatisticamente significantes. Houve diferenças nos padrões de distribuição semanal dos pacientes do SUS (4.120), dos sistemas de prépagamento (1.225) e dos particulares (459). Para os pacientes do SUS e dos sistemas de pré-pagamento o número de hospitalizações foi elevado no início da semana e houve uma diminuição gradual no restante dos dias. Para os particulares, o número de hospitalizações foi baixa no domingo e houve elevação na sexta e sábado. Não houve associação com o sexo ou com a condição de saída.

\begin{abstract}
Introduction

There is in the literature some studies looking for cycles and a circadian rhythm associated with acute myocardial infarction. Several authors have pointed out a higher incidence of myocardial infarction on Mondays suggesting the existence of trigger mechanisms in the disease onset. The week day distribution of hospitalizations due to
\end{abstract}


myocardial infarction and its associations with patient sex and category of hospitalization (social status) in the Ribeirão Preto area from 1987 to 1996, were studied.

\section{Methods}

A total of 173,982 hospitalizations due to heart and cerebral vascular diseases, including 5,804 cases of acute myocardial infarction, were evaluated using the medical records of the Hospital Data Center of the medical school of Ribeirão Preto, University of S. Paulo. The hospitalizations were classified as being on a private basis, through private insurance or the Unified Health System (SUS). The associations with sex, patient condition at discharge and category of the hospitalization were studied.

\section{Results/Conclusions}

The distribution of hospitalizations due to heart and vascular diseases and acute myocardial infarction showed a peak incidence on Mondays, corresponding to $19.3 \%$ and $16.9 \%$ of the cases. During the weekend there was a decrease in the number of heart and vascular disease hospitalizations (8.6\%) and a smaller reduction in the number of myocardial infarction cases (12.7\%) which are statistically significant. In addition, there were observed differences in the distribution patterns according to the day of the week and category of hospitalization. While the SUS patients $(4,120)$ and private insurance patients $(1,225)$ are more likely to be hospitalized on Mondays, private patients (459) are more likely to be hospitalized on Fridays and Saturdays. There were no associations with patient sex and patient condition at discharge.

\section{INTRO DU ÇÃO}

O infarto do miocárdio é um dos diagnósticos mais freqüentes nos pacientes hospitalizados nos países ocidentais. ${ }^{6}$ A despeito do grande acúmulo de conhecimentos acerca da etiologia, fisiopatologia, epidemiologia, história natural, diagnóstico e tratamento das doenças cardiovasculares, nos últimos 50 anos, elas continuam sendo o mais importante grupo de causas de morbidade e mortalidade a partir da quarta década de vida. Entre elas, destacam-se a doença arteriosclerótica coronariana e o infarto do miocárdio e seus conhecidos fatores de risco: idade, sexo, hipercolesterolemia, hipertensão, diabetes mellitus, obesidade, hábito de fumar, vida sedentária, estresse psicológico e antecedentes familiares, entre outros.

A investigação de fatores biológicos associados à doença coronariana e ao infarto privilegiou-os na interpretação da determinação da doença, com a criação de modelos explicativos, nos quais o papel dos lipídios representa a idéia dominante acerca da causa da aterogênese e das doenças isquêmicas do coração. As mudanças no estilo de vida a ela relacionadas seriam as responsáveis pelas alterações no padrão de mortalidade pelas doenças isquêmicas do coração. ${ }^{1}$ De outro lado, a investigação da associação de fatores extrabiológicos, principalmente as condições de vida e de trabalho, estreitamente associados à posição social do indivíduo, levou à criação de modelos sócio-históricos do fenômeno da saúde e da doença. Yazlle Rocha et al ${ }^{9}$ discutiram as desigualdades entre pacientes hospitalizados por doenças cardíacas e vasculares cerebrais assinalando a associação das mesmas com a posição social dos indivíduos e a sua relação com os sistemas sociais de assis- tência médica. Encontraram que os grupos sociais menos privilegiados economicamente são hospitalizados a uma idade média 10 anos menor. Também, a idade média ao morrer dos pacientes hospitalizados é mais precoce entre os menos privilegiados, fato esse que também ocorre em outros tipos de patologias. ${ }^{7,11}$

A procura de variações temporais - gerais, sazonais ou cíclicas - no fenômeno do infarto tem interesse do ponto de vista da elucidação dos fatores causais, bem como do ponto de vista da assistência e planejamento em saúde. São interessantes os estudos cronobiológicos que buscam variações sazonais, dia da semana ou hora do dia em que mais frequientemente ocorre o infarto. Como referido por Choisnel et al, ${ }^{2}$ na população em geral há um certo número de pessoas em risco de sofrer infarto do miocárdio a qualquer momento. É lembrado que o ataque do infarto do miocárdio é um processo fisiopatológico muito complexo, no qual muitos fatores jogam cada um o seu papel - idade, sexo, nutrição, tabagismo e outros -, no meio dos quais a mudança atmosférica - por ele estudada - responderia por $10 \%$ dos casos nessas situações.

O presente trabalho tem por objetivo estudar a variação semanal das hospitalizações por infarto do miocárdio e sua associação ao sexo e à categoria da internação (condição social), na região de Ribeirão Preto, de 1987 a 1996. Para tanto, foram levadas em consideração as condições especiais existentes na região: número de leitos hospitalares suficientes, múltiplos sistemas de financiamento das hospitalizações, estabelecimentos de saúde especializados de nível secundário e terciário e população bem definida, o que leva a admitir que a quase totalidade da demanda regional de internações é atendida na própria região. ${ }^{3}$ De outro lado, a existência de 
quarto do valor do pico). Esta distribuição desigual ao longo da semana é significativa estatisticamente.

Tabela 1 - Hospitalização por doenças cardíacas e vasculares cerebrais (DCVC) e infarto do miocárdio (IM), região de Ribeirão Preto, 1987-1996.

\begin{tabular}{lccl}
\hline Ano & DCVC & IM & Total \\
\hline 1987 & 12.205 & 444 & 12.649 \\
1988 & 15.739 & 536 & 16.275 \\
1989 & 16.117 & 546 & 16.663 \\
1990 & 15.642 & 539 & 16.181 \\
1991 & 16.522 & 566 & 17.088 \\
1992 & 17.885 & 678 & 18.563 \\
1993 & 19.054 & 630 & 19.684 \\
1994 & 19.608 & 604 & 20.212 \\
1995 & 18.111 & 641 & 18.752 \\
1996 & 17.295 & 620 & 17.915 \\
\hline Total & 168.178 & 5.804 & 173.982 \\
\hline
\end{tabular}

Fonte: Centro de Processamento de Dados Hospitalares da Faculdade de Medicina de Ribeirão Preto da USP (CPDH/D M S/FM RP) de planos de saúde, medicina de grupo, cooperativas, convênios ou de autogestão) e SUS (sistema público).

Nesse último incluem-se as internações no único hospital geral público, e na sua unidade de emergência e também os pacientes assistidos em hospitais privados conveniados ou contratados pelo SUS. Essas categorias de hospitalização representam a posição social diferenciada e um gradiente de custeio e assistência decrescente: nível elevado para os particulares (principalmente trabalhadores não manuais) e nível mínimo para o sistema público (principalmente trabalhadores manuais). ${ }^{11}$ As outras variáveis estudadas foram: data da internação - transformada em dia da semana - sexo e condição de saída do paciente.

Para processamento dos dados foram utilizados o Epi Info 6.04 e o Dbase IV. A associação entre as variáveis foi testada pelo $\mathrm{X}^{2}$ com nível de significância de 5\%.

\section{RESU LTADOS}

Na Tabela 1 verificou-se que, embora os dados apresentem oscilações, há tendência crescente no número de internações - com pico de 20.212 casos em 1994 , como também nos casos de infarto - com pico de 678 casos em 1992.

Na Tabela 2 observou-se que para as doenças cardíacas há um pico de hospitalização às segundas-feiras $(19,3 \%)$ com gradual queda na freqüência nos dias seguintes, apresentando redução a menos da metade do valor do pico no sábado e no domingo (8,2\% e 8,6\%). Com as hospitalizações por infarto agudo do miocárdio ocorre padrão semelhante - pico na segunda-feira $(16,9 \%)$ e redução no sábado e no domingo $(12,6 \%$ e $12,7 \%$ ), porém a redução é muito menor (em torno de um
Tabela 2 - Hospitalização por doenças cardíacas e vasculares cerebrais e infarto do miocárdio segundo o dia da semana, região de Ribeirão Preto, 1987-1996.

\begin{tabular}{lrrrrrrr}
\hline \multirow{2}{*}{ Dia da semana } & \multicolumn{2}{c}{ O ut. DCVC } & \multicolumn{2}{c}{ Infarto } & \multicolumn{2}{c}{ Total } \\
& $N$ & \%* & N & \%* & N & \%* \\
\hline Domingo & 14.490 & 8,6 & 737 & 12,7 & 15.227 & 8,8 \\
Segunda & 32.534 & 19,3 & 981 & 16,9 & 33.515 & 19,3 \\
Terça & 29.378 & 17,5 & 840 & 14,5 & 30.218 & 17,4 \\
Quarta & 27.888 & 16,6 & 866 & 14,9 & 28.754 & 16,5 \\
Quinta & 26.958 & 16,0 & 841 & 14,5 & 27.799 & 16,0 \\
Sexta & 23.126 & 13,8 & 805 & 13,9 & 23.931 & 13,8 \\
Sábado & 13.804 & 8,2 & 734 & 12,6 & 14.538 & 8,4 \\
\hline Total** & 168.178 & 96,7 & 5.804 & 3,3 & 173.982 & 100,0 \\
\hline
\end{tabular}

Fonte: CPDH/ DMS/FMRP

* Percentual na coluna

**Percentual em relação ao total geral

$X^{2}=303,22 \quad$ g.l. $=6 \quad p=0,0000000$

A análise segundo o dia da semana e sexo (Tabela 3) e a condição do paciente na saída do hospital (Tabela 4), mostrou que $67,6 \%$ dos casos de infarto ocorreram no sexo masculino e $32,4 \%$ no feminino; $81 \%$ dos casos receberam alta e $19 \%$ terminaram em óbito; em relação à distribuição semanal manteve-se o padrão antes descrito, porém não há associação estatística significativa entre os dias da semana e o sexo ou condição de saída.

Quanto à distribuição das hospitalizações por infarto agudo do miocárdio, segundo o dia da semana e a categoria da hospitalização (Tabela 5), 7,9\% dos casos ocorreram em pacientes particulares, $21,1 \% \mathrm{em}$ pacientes de sistemas privados de pré-pagamento e $71 \%$ em pacientes do SUS (aí incluídos os pacientes do sistema previdenciário público anterior à criação do SUS). Os pacientes do SUS e dos sistemas de pré- 
Tabela 3 - Hospitalização por infarto segundo dia da semana e sexo, região de Ribeirão Preto, 1987-1996.

\begin{tabular}{|c|c|c|c|c|c|c|}
\hline \multirow[t]{2}{*}{ Dia } & \multicolumn{2}{|c|}{ Masculino } & \multicolumn{2}{|c|}{ Feminino } & \multicolumn{2}{|c|}{ Total } \\
\hline & $\mathrm{N}$ & $\% *$ & $\mathrm{~N}$ & $\% *$ & $\mathrm{~N}$ & $\% *$ \\
\hline $\begin{array}{l}\text { Domingo } \\
\text { Segunda } \\
\text { Terça } \\
\text { Quarta } \\
\text { Quinta } \\
\text { Sexta } \\
\text { Sábado }\end{array}$ & $\begin{array}{l}506 \\
666 \\
556 \\
595 \\
550 \\
536 \\
514\end{array}$ & $\begin{array}{l}12,9 \\
17,0 \\
14,2 \\
15,2 \\
14,0 \\
13,7 \\
13,1\end{array}$ & $\begin{array}{l}231 \\
315 \\
284 \\
271 \\
291 \\
269 \\
220\end{array}$ & $\begin{array}{l}12,3 \\
16,7 \\
15,1 \\
14,4 \\
15,5 \\
14,3 \\
11,7\end{array}$ & $\begin{array}{l}737 \\
981 \\
840 \\
866 \\
841 \\
805 \\
734\end{array}$ & $\begin{array}{l}12,7 \\
16,9 \\
14,5 \\
14,9 \\
14,5 \\
13,9 \\
12,6\end{array}$ \\
\hline Total** & 3.923 & 67,6 & 1.881 & 32,4 & 5.804 & 100,0 \\
\hline
\end{tabular}

Tabela 4 - Distribuição semanal dos casos de infarto segundo condição de saída - região de Ribeirão Preto, 1987-1996.

\begin{tabular}{lrrrrrr}
\hline Dia & \multicolumn{2}{c}{ Altas } & \multicolumn{2}{c}{ Óbitos } & \multicolumn{2}{c}{ Total } \\
& $\mathrm{N}$ & \%* & $\mathrm{N}$ & $\% *$ & $\mathrm{~N}$ & \%* \\
\hline Domingo & 583 & 12,4 & 154 & 14,0 & 737 & 12,7 \\
Segunda & 802 & 17,1 & 179 & 16,2 & 981 & 16,9 \\
Terça & 705 & 15,0 & 135 & 12,2 & 840 & 14,5 \\
Quarta & 693 & 14,7 & 173 & 15,7 & 866 & 14,9 \\
Quinta & 680 & 14,5 & 161 & 14,6 & 841 & 14,5 \\
Sexta & 642 & 13,7 & 163 & 14,8 & 805 & 13,9 \\
Sábado & 596 & 12,7 & 138 & 12,5 & 734 & 12,6 \\
\hline Total & 4.701 & 81,0 & 1.103 & 19,0 & 5.804 & 100,0 \\
\hline
\end{tabular}

Fonte: CPDH/ DMS/ FMRP

*Percentual na coluna

**Percentual em relação ao total geral

$X^{2}=7,71 \quad$ g.l. $=6 \quad p=0,22770428$

pagamento apresentaram variação semanal com pico de freqüência às segundas-feiras $(17,4 \%$ e 16,2\%) e tendência à redução no restante da semana. Os pacientes particulares apresentaram distribuição semanal diferente, com menor freqüência no domingo (10,5\%), elevação ao longo da semana e pico no sábado $(17,6 \%)$. Assim, a variação semanal da frequiência de hospitalizações por infarto agudo do miocárdio encontrada é decrescente de segunda a domingo nos pacientes do SUS e de pré-pagamento e crescente de domingo a sábado nos pacientes particulares. Há associação estatisticamente significante entre o dia da semana e as categorias de internação.

\section{DISCU SSÃO}

A assistência hospitalar é o recurso reservado a pacientes que não podem ser adequadamente assistidos em outros níveis ou tipos de serviços, geralmente por necessidade terapêutica e/ou diagnóstica. Além disso, a assistência hospitalar é altamente seletiva, tanto tecnicamente - no que diz respeito à seleção dos casos segundo a sua complexidade e à existência de recursos adequados -, como economicamente - no
Tabela 5 - Hospitalização por infarto segundo dia da semana e categoria da internação, região de Ribeirão Preto, 1987-1996.

\begin{tabular}{|c|c|c|c|c|c|c|c|c|}
\hline \multirow[t]{2}{*}{$\overline{\mathrm{Dia}}$} & \multicolumn{2}{|c|}{ Particular } & \multicolumn{2}{|c|}{ Pré-pagam. } & \multicolumn{2}{|c|}{ SUS } & \multicolumn{2}{|c|}{ Total } \\
\hline & $\mathrm{N}$ & & $\mathrm{N}$ & & $\mathrm{N}$ & $\% *$ & $\mathrm{~N}$ & $\% *$ \\
\hline $\begin{array}{l}\text { Domingo } \\
\text { Segunda } \\
\text { Terça } \\
\text { Q uarta } \\
\text { Quinta } \\
\text { Sexta } \\
\text { Sábado }\end{array}$ & $\begin{array}{l}048 \\
66 \\
65 \\
52 \\
71 \\
76 \\
81\end{array}$ & $\begin{array}{l}10,5 \\
14,4 \\
14,2 \\
11,3 \\
15,5 \\
16,6 \\
17,6\end{array}$ & $\begin{array}{l}149 \\
198 \\
193 \\
183 \\
178 \\
172 \\
152\end{array}$ & $\begin{array}{l}12,2 \\
16,2 \\
15,8 \\
14,9 \\
14,5 \\
14,0 \\
12,4\end{array}$ & $\begin{array}{l}540 \\
717 \\
582 \\
631 \\
592 \\
557 \\
501\end{array}$ & $\begin{array}{l}13,1 \\
17,4 \\
14,1 \\
15,3 \\
14,4 \\
13,5 \\
12,2\end{array}$ & $\begin{array}{l}737 \\
981 \\
840 \\
866 \\
841 \\
805 \\
734\end{array}$ & $\begin{array}{l}12,7 \\
16,9 \\
14,5 \\
14,9 \\
14,5 \\
13,9 \\
12,6\end{array}$ \\
\hline Total** & 459 & 7,9 & .225 & 21,1 & 4.120 & 71,0 & 5.804 & 100,0 \\
\hline \multicolumn{9}{|c|}{$\begin{array}{l}\text { Fonte: CPDH/DMS/FM RP } \\
\text { SUS - Sistema Ú nico de Saúde } \\
* \text { Percentual na coluna } \\
* * \text { Percentual em relação ao tota } \\
X^{2}=24,64 \quad \text { g.l. }=12 \quad p=0,(\end{array}$} \\
\hline
\end{tabular}

que diz respeito à cobertura das despesas decorrentes da assistência, diretamente pelo paciente ou indiretamente por sistemas públicos ou privados.

Quanto à decisão do momento da hospitalização, pode-se dizer que a maioria dos casos é de procedimentos eletivos, isto é, não correndo risco de vida as pessoas podem escolher o melhor momento para afastar-se dos seus afazeres e submeter-se por alguns dias aos cuidados de que carecem. Os casos com risco de vida - emergências - devem, no geral, ser transportados imediatamente para unidades especiais - prontosocorros e emergências - em hospitais, onde podem receber rapidamente a melhor assistência possível aos seus problemas. Entre os problemas de saúde - exceto os traumatismos e acidentes - poucos são tão urgentes e dramáticos como o infarto agudo do miocárdio. A dor é a queixa inicial mais comum nos pacientes com infarto do miocárdio. Em alguns casos o desconforto é suficientemente intenso para ser descrito como a pior dor que o paciente jamais sentiu. ${ }^{6}$ Isso faz com que esse desconforto não passe despercebido e leve o paciente a procurar auxílio imediatamente. Não foram encontrados dados sobre o percentual de pacientes que morrem sem ter tido chance de procurar assistência. Constatado o infarto, a conduta é, geralmente, a hospitalização, que se efetivará até algumas horas após o início dos sintomas. Para que isso tudo ocorra deve haver disponibilidade de especialistas e serviços com razoável distribuição espacial e acessibilidade do ponto de vista da cobertura financeira. Essas condições existem para a maior parte da população residente na região de Ribeirão Preto. ${ }^{3}$ Isso posto, pode-se dizer que o dia da internação por infarto agudo do miocárdio na região de Ribeirão Preto corresponde, na grande maioria dos casos, ao mesmo dia do início dos sintomas e que, portanto, a distribuição semanal das hospitalizações corresponde à distribuição da ocorrência dos casos de infarto. 
como o dia de pico do infarto, o que estaria associado à mudança de um período de atividades livres para outro de trabalho programado. Nos presentes achados, o risco atribuível ao fator segunda-feira no infarto do miocárdio é de $18 \%$ acima da média e se reduz a $12 \%$ abaixo no final de semana.

No presente estudo, a variação semanal das hospitalizações por infarto agudo do miocárdio não apresenta associação estatística significante nem com o sexo nem com a condição no egresso de alta ou óbito (Tabelas 3 e 4). É significativa a associação encontrada entre a variação semanal e a categoria da internação - que reflete a condição social do paciente. Os dados são compatíveis com os descritos por Willich et al, ${ }^{8}$ onde a segunda-feira é o dia de maior risco para o infarto na população de trabalhadores manuais (blue collar) e o final de semana para os trabalhadores não manuais (white collar). Utilizando a categoria da internação como indicador da condição social dos pacientes, ${ }^{11}$ observa-se que os usuários do SUS e dos sistemas de pré-pagamento são majoritariamente trabalhadores manuais e apresentam risco atribuível ao fator segunda-feira de $22 \%$ a $13 \%$ acima da média semanal, respectivamente. Entre os pacientes particulares se concentram os profissionais, administradores e técnicos de nível superior - trabalhadores não manuais -, que no presente estudo apresentam tendência crescente de hospitalizações por infarto com pico de freqüência no sábado e risco atribuível de $23 \%$ acima da média semanal. O "gatilho" para os pacientes particulares - ao contrário dos trabalhadores, para quem ele está centrado no início da semana de trabalho - estaria representado pelo abandono da posição social no trabalho e/ou pelo retorno às atividades no lar e ao lazer no sábado, ilustrando mais uma característica da desigualdade social diante da doença. ${ }^{?}$

\section{AG RADECIMENTOS}

A Lourenço Gallo Jr., docente do Departamento de Clínica Médica da Faculdade de Medicina de Ribeirão Preto, USP, pelas indicações sugeridas.

\section{REFERÊNCIAS}

1. Azambuja MIR. Rise and fall in ischemic heart disease mortality: it may have happened before. Rev Saúde Pública 1995;29:440-3.

2. Choisnel J, Cohen JCl, Poisvert M, Thournout A. Weather and acute cardiovascular attacks: statistical analysis and results. Experientia 1987;(43):27-32.

3. D'Oleo RJM. U tilização dos leitos hospitalares na região de Ribeirão Preto, 1988 [dissertação]. Ribeirão Preto: Faculdade de Saúde Pública da USP; 1991.

4. Fávero M, Yazlle Rocha JS, Haddad N, Teruel JR O rganização de um Centro de Informática Hospitalar em nível local. Rev Paul Hosp 1973;(21):151-7.

5. Gnecchi-Ruscone T, Piccaluga E, Guzzetti S, Contini M, Montano N, Nicolis E. Morning and Monday: critical periods for the onset of acute myocardial infarction. The GISSI 2 Study Experience. Eur Heart J 1994;15:882-7.

6. Pasternak CR, Braunwald E. Infarto agudo do miocárido. In: Isselbacher K, Braunwald E, Wilson J, Martin J, Fauci A, Kasper D. Harrison - Medicina interna. 13aㅡ ed. México: Mc Graw-Hill; 1994. p. 1116-27. 
7. Whitehead $M$. The health divide. In: Townsend $P$ Davidson N. Inequalities in health: the Black Report London: Penguin Books; 1992.

8. Willich SN, Lowel H, Lewis M, Hormann A, Arntz $H R$, Keil, U. Weekly variation of acute myocardial infarction. Increased Monday risk in the working population. Circulation 1994;90:87-93.

9. Yazlle Rocha JS, Jorge AO, Simões BJG, Vichi FL. Desigualdades entre pacientes hospitalizados por doenças cardíacas e vasculares cerebrais em localidade do Estado de São Paulo (Brasil), 1986. Rev Saúde Pública 1989;23:374-81.
10. Yazlle Rocha JS, Simões BJG, Forster AC. Estudos de demanda e utilização de serviços de saúde: uma experiência regional. Medicina (Ribeirão Preto) 1993;26:636-42.

11. Yazlle Rocha JS, Simões BJG, Guedes GLM. Assistência hospitalar como indicador da desigualdade social. Rev Saúde Pública 1997;31:479-87.

12. Yazlle Rocha JS, Simões BJG. Assistência hospitalar pública e privada em bases populacionais, 19861996. Rev Saúde Pública 1999;33:44-54 